\title{
Photovoltaic Sensor for the Solar Battery Guidance on the Sun Based on GeS:Sb Layered Crystals
}

\author{
D. I. Bletskan1, V. M. Kabatsii' ${ }^{2}$, M. M. Bletskan1 \\ ${ }^{1}$ Department of Physics, Uzhgorod National University, Uzhgorod, Ukraine \\ ${ }^{2}$ Mukachevo State University, Mukachevo, Ukraine \\ Email: crystal_lab457@yahoo.com
}

Received 14 February 2016; accepted 28 March 2016; published 31 March 2016

Copyright (C) 2016 by authors and Scientific Research Publishing Inc.

This work is licensed under the Creative Commons Attribution International License (CC BY). http://creativecommons.org/licenses/by/4.0/

cC) (i) Open Access

\begin{abstract}
Photovoltaic tracking sensor monitoring the position of the sun in the sky was developed on the basis of GeS:Sb layered crystals. The operating principle of this sensor is based on the phenomenon of photovoltaic effect in GeS:Sb crystals; the magnitude and sign of generated photo-emf depend on the position of the light probe relative to the ohmic contacts, deposited on (001) surface of the crystal.
\end{abstract}

\section{Keywords}

Photovoltaic Sensor, Photo-Emf, Germanium Monosulfide, Solar Tracking

\section{Introduction}

The improvement of manufacturing techniques of solar power plants and the increasing of efficiency of photovoltaic panels is an actual problem in the field of alternative energy. The maximum energy generated by solar modules is obtained in the case of normal incidence of the sun's rays on the plane of modules. Therefore, to increase the efficiency of solar modules is necessary to provide the automatic tracking of solar modules behind the sun. A variety automated orientation systems of solar modules are used for these purposes as the incidence angle of sun's rays on solar modules as well as the direction of movement of the sun across the sky [1]-[3]. With automatic sun tracking maintain precise guidance of solar modules by measuring of photo-emf or current in photocells of the sun position sensors and moving them using the rotating mechanism of both coordinates. The photovoltaic tracking sensors of the sun position in the sky are the important elements of the electronic control circuit of angular or linear movement of rotary modules. In this paper, the possibility of use and the design of pho- 
tovoltaic sensors developed on the basis of germanium monosulfide crystals doped by antimony for the tracking the position of the sun in the sky as well as for non-contact inertialess measurements of angular or linear movements with regard to their directions are described.

\section{Preparation of Crystals}

A special feature of germanium monosulfide is the one-sided homogeneity region [4]. Therefore, regardless of the growth method, the GeS crystals always grow with a high concentration of cation vacancies $\left(10^{17}-10^{18} \mathrm{~cm}^{-3}\right)$ and they are $p$-type of conductivity, low-resistance and poorly photosensitive. Antimony doping of germanium monosulfide leads to the "healing" of cation vacancies and, consequently, is accompanied by an increase in specific dark resistance and the integral photosensitivity.

Germanium monosulfide crystals doped with Sb were grown by the static sublimation method in the sealed pre-evacuated up to 133 Pa quartz ampules 18 - $22 \mathrm{~mm}$ in diameter and 180 - $200 \mathrm{~mm}$ in length. Synthesis of initial polycrystalline substance GeS was carried out by direct fusion of elementary germanium and sulfur, taken in a stoichiometric ratio. For doped crystals in the initial charge was added antimony in an amount of 0.1 and 1.0 at. \%, and the synthesis was carried out with the impurity. Upon synthesis completion the received polycrystalline ingot was shaken into one end of the ampoule, which then was placed in a horizontal two-zone tubular furnace of resistive heating. The optimum crystal growing conditions are following: the temperature of the evaporation zone is $900 \mathrm{~K}$; the temperature of the condensation zone is $800 \mathrm{~K}$.

The crystals from the gas phase were grown in the form of plane-parallel plates in the sizes of $8 \times 15 \times 01$ $\mathrm{mm}$. The layered character of structure explains the lamellar habit of crystals with well-developed (001) faces.

For carrying out electrical and photoelectric measurements the coplanar contacts were deposited on natural crystal (001) faces by an indium fusing such a way that electric field was directed along the crystallographic axis $b$. According to thermo-emf sign it was established that specially undoped and antimony doped GeS crystals had the $p$-type conductivity.

\section{Photo-Emf in GeS:Sb Crystals}

In GeS:Sb crystals it is observed the bulk photo-emf in the absence of offset at the shielded contacts. The absolute value of photo-emf is $\sim 200 \mathrm{mkV}$. The longitudinal mode was used in the measurements with the contacts deposited on (001) plane. The light probe width did not exceed $0.5 \mathrm{~mm}$. The ohmic character of contacts was confirmed by linear current-voltage characteristic as with the light as well as in the dark. The spectral distribution of photo-emf for various light probe positions with respect to the ohmic contacts is shown in Figure 1.

From a comparison of photo-emf (Figure 1(a), curves 1 and 3) and photoconductivity (Figure 1(a), curve 2) spectra measured on the same GeS:Sb crystal it is visible that additional maxima and features on the spectral curves measured without the application of external electric field are not observed. Moreover, the maxima of spectral distribution of photo-emf are at the same energies that the maxima of intrinsic photoconductivity (PC), i.e. in the fundamental absorption edge field of germanium monosulfide. Thus, for the studied crystals it is not found the contribution of photoionization of impurity atoms in the photo-emf formation at both room and low temperatures. Hence, the photo-emf in GeS:Sb crystal volume arises as the results of generation of electron-hole

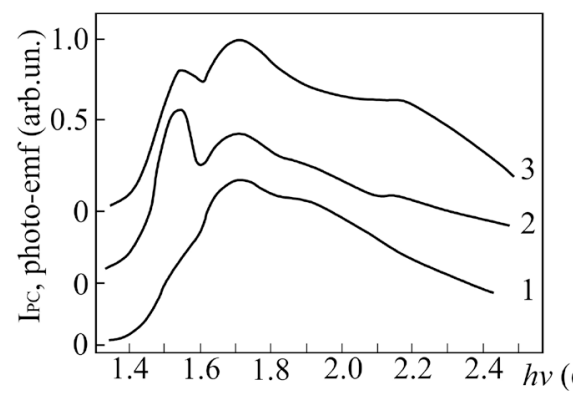

(a)

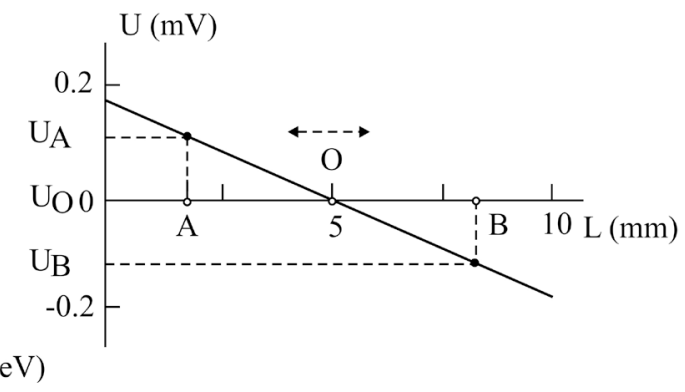

(b)

Figure 1. (a) Photo-emf spectra measured at two light probe positions (curve 1-at A point, curve 3-at B point) and photoconductivity spectrum (2) of GeS:Sb crystal; (b) The probe characteristic of photo-emf effect. 
pairs by light from the intrinsic absorption region with their subsequent spatial separation.

The character of the long-wavelength range of photo-emf spectrum is defined by the intrinsic absorption and it is not detected the contribution of impurity centers in the photo-emf formation at least at the room temperature. The lack of long-wave tail indicates on the small probability of injection from electrodes into the semiconductor in this case. There is a significant difference in a short-wave part of photo-emf and photoconductivity spectra can be caused by uneven distribution of electric field in the thickness of the sample.

According to modern concepts the photovoltaic effect in semiconductors is based on the phenomenon of spatial separation of the nonequilibrium electron-hole pairs generated by light. Light absorption and photoionization only increase the number of free electrons and holes, but do not separate them in the space. The appearance of the photovoltaic effect requires additional reasons for the separation of nonequilibrium charges of different signs. Any inhomogeneity in the crystal volume can lead to the photo-emf generation. Such inhomogeneity can be created by the impurity fluctuations in a sample or by the second phase inclusions. The photovoltaic phenomena are characteristic for inhomogeneous samples as a result of modulation of intrinsic potential barriers. In our case the most probable cause of generation of bulk photo-emf is inhomogeneous distribution of compensating antimony impurity and structural defect-complexes $\left\{\mathrm{V}_{\mathrm{Ge}}-\mathrm{Sb}_{\mathrm{Ge}}\right\}$. This defect represents the inhomogeneous distribution of charged centers forming the cluster accumulations, which creates significant potential barriers in the crystal volume, separating the redundant (nonequilibrium) charge carriers by the electric field. The observable decreasing of photo-emf signal along with the sample temperature increasing is due to the decreasing of volume and height of potential barriers as well as due to the strengthening of thermal ionization of the centers.

We found a number of interesting features under the study of photovoltaic effect in the metal- $\mathrm{GeS}: \mathrm{Sb}-$ metal structure. In particular, it is found that the light probe position relative to the ohmic contacts significantly effect on the general view of spectral dependence of short circuit current of photovoltaic effect (curves 1, 3 in Figure 1(a)). The significant spectra transformation are observed near the maximum (1.52 eV), which corresponds to the fundamental absorption edge of germanium monosulfide doped by antimony. Furthermore, in the case of longitudinal photovoltaic effect in GeS:Sb layered crystals, i.e. when the ohmic contacts are deposited on (001) surface, the photo-emf reaches a maximum when the light probe location close to one of contacts and decreases uniformly while moving in the direction toward other contact, taking (reaching) a value of zero when the light probe reaches the middle of distance between the contacts (point $\mathrm{O}$ in Figure 1(b)). Further movement of the light probe in the direction toward other contact is accompanied by inversion of photo-emf sign and increasing of its magnitude to the maximum value near the second contact. This feature of the probe characteristic of photo-emf effect in GeS:Sb single crystals we used for creation of photoelectric sensors of linear and angular movement [5]-[10].

\section{Photoelectric Sensor for the Rotary Mechanism of Solar Energy Installation}

The basic element of the photoelectric sensor of angular and linear movement of solar batteries is an active element (Figure 2(a)), which consists from a frame 1, at its bottom there is placed the fluoroplastic substrate with the attached plane-parallel plate 2 of GeS:Sb crystals with the deposited ohmic indium contacts 4 connected to the current-outputs 5 . The frame is hermetically closed from above by the window 3 which is transparent for the sunlight. The dependence of signal value on the amplifier input from the light probe location relative to the ohmic contacts of active elements is defined by the probe characteristic (Figure 1(b)).

The functional scheme of photoelectric sensor for the rotary module of solar batteries is shown in Figure 3 . Two independent active elements 1, 2 are fixed respectively in horizontal and vertical positions and they are in focuses of parabolic mirrors 3,4 . The electrical signals from active elements 1,2 are sent to the processing block of electrical signals 5 which comprises the amplifiers 6,7 , an analog-digital converter 8 and the microprocessor 9 which also controls the operation of amplifiers.

The photoelectric sensor for the rotary module of solar batteries works as follows. Initially the parabolic mirrors are adjusted such a way that sun's rays reflected from them then fall exactly on the middle of active elements 1, 2 (point $\mathrm{O}$ in Figure 1(b)). The photo-emf is equal to zero and the signal in a circuit is absent at such arrangement of a light probe on GeS:Sb crystal surface, as follows from Figure 1(b). The change of Sun location on the firmament leads to the movement of the light rays reflected from parabolic mirrors in the direction to one of the contacts 4 (Figure 2(a)) along active elements 1, 2 (for example into A point or B point in Figure 1(b)), that as a result leads to the photo-emf generation which arrives on inputs of amplifiers 6, 7 (Figure 3). The voltage magnitude and polarity on outputs of amplifiers 6,7 are given to the analog-digital converter 8 


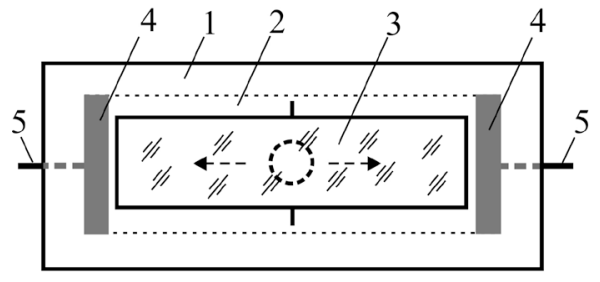

(a)

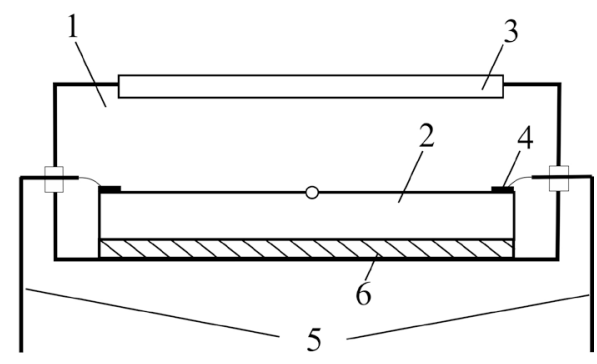

(b)

Figure 2. The construction of an active element of photoelectric sensor: top view (a) and side view (b); 1 -frame; 2 plane-parallel plate of GeS:Sb; 3-transparent window; 4-ohmic contacts; 5-current-outputs, 6-fluoroplastic substrate.

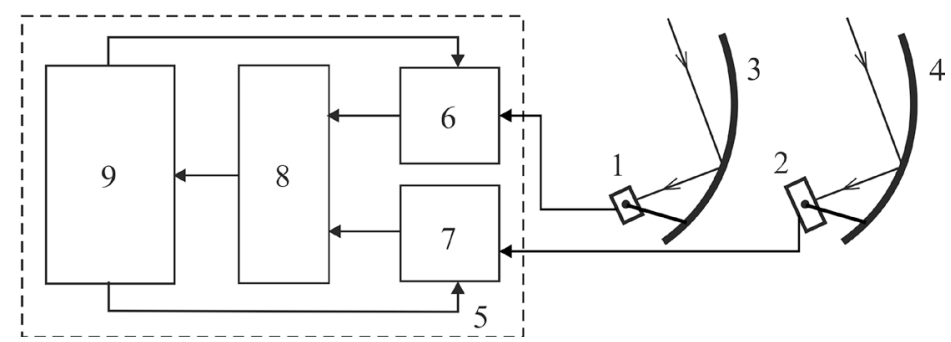

Figure 3. The functional scheme of photoelectric sensor of the rotary module of solar batteries: 1, 2-active elements; 3, 4parabolic mirrors; 5-processing block of electric signals; 6, 7-amplifiers; 8-analog-digital converter; 9-microprocessor.

which generates the corresponding digital codes for the microprocessor 9. After processing of received digital codes the microprocessor 9 sends the corresponding electrical control signals on the rotary modules with the aim of rotate of solar batteries to the required direction. When the voltage drop on the inputs of amplifiers 6,7 becomes equal to zero, then the microprocessor 9 passes to a timer mode that provides the energy efficiency of photoelectric sensor. The operation of microprocessor 9 with varying periodicity and time duration can be set by hardware or software depending on the chosen type of rotary modules and their operation conditions.

\section{The Photoelectric Sensor for Measurements of Angular or Linear Movements}

The described above construction of active element (Figure 2) manufactured on the basis of GeS:Sb layered crystals we used for creation of the non-contact photoelectric sensor for measurements of angular or linear movements which functional scheme is shown in Figure 4.

The photoelectric sensor of angular or linear movements works as follows. Initially the light beam is routed from the radiation source 6 to the convex mirror 8 , where it reflects and falls on the middle of active element (O point in Figure 1(b)). In this case the voltage of output of amplifier 3 is equal to zero $\left(U_{\mathrm{O}}=0 \mathrm{~V}\right)$ and it is taken as reference. The analog-digital converter 4 generates a corresponding digital code for entering it into the memory of microprocessor 6 . The angular or linear movement of mirror 8 leads to the shift of light beam along active element (for example from $\mathrm{O}$ point to A point in Figure 1(b)) and to photo-emf generation. At the same time the microprocessor 5 starts the measurement process of $U_{\mathrm{A}}$ voltage magnitude and polarity on the output of amplifier 3 which is transmitted to the analog-digital converter 4 . As a result of transformations, the analog-digital converter 4 generates the corresponding digital code for recording it into the memory of microprocessor 5 . The changing of voltage polarity allows to determine the direction of angular or linear movement, and the difference in values of $U_{\mathrm{A}}$ and $U_{\mathrm{O}}$ voltages proportional corresponds to the magnitude of angular or linear movement. The microprocessor 5 chooses the magnitude of the previous value of $U_{\mathrm{A}}$ voltage as the value of reference voltage in the case of necessity to continue the measurements relative to the last movement of a mirror 8 . If in the generating process of a light ray by the radiation source 6 there was no change of position of angular or linear movement mechanism 7, then the voltage on an input of amplifier 3 is equal zero and the microprocessor 5 passes into the timer mode, and the radiation source 6 continues to run independently until the photo-emf occurrence at the input of amplifier 3 . The operation of microprocessor 5 with different periodicity and time duration can be set by hardware or software. 


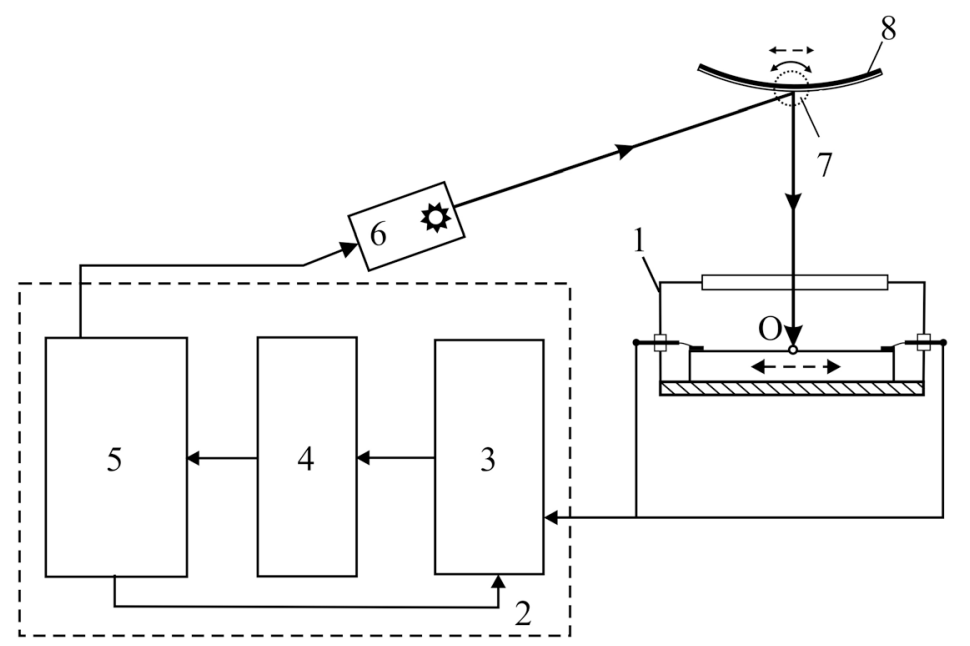

Figure 4. Functional scheme of the photoelectric sensor of angular or linear movements: 1—active element; 2-processing block of electrical signals; 3-amplifier; 4-analog-digital converter; 5-microprocessor; 6—radiation source; 7—mechanism of angular or linear movement; 8-convex mirror.

\section{Conclusion}

Thus, the revealed phenomenon of photovoltaic effect and the unusual probe characteristic of photo-emf in $\mathrm{GeS}: \mathrm{Sb}$ crystals were used for creation of photoelectric sensors for automatic tracking of the Sun location on the firmament as well as for the angular or linear movement. It described the tracking system behind the Sun on the firmament using the photovoltaic sensor (PVS) for photovoltaic modules (PVM) and energy systems.

\section{References}

[1] Camacho, E.F., Berenguel, M., Rubio, F.R. and Martinez, D. (2012) Control of Solar Energy Systems. Springer-Verlag, London. http://dx.doi.org/10.1007/978-0-85729-916-1

[2] Seme, S. and Štumberger, G. (2011) A Novel Prediction Algorithm for Solar Angles Using Solar Radiation and Differential Evolution for Dual-Axis Sun Tracking Purposes. Solar Energy, 85, 2757-2770. http://dx.doi.org/10.1016/j.solener.2011.08.031

[3] Bazyari, S., Keypour, R., Farhangi, S., Ghaedi, A. and Bazyari, K. (2014) A Study on the Effects of Solar Tracking Systems on the Performance of Photovoltaic Power Plants. Journal of Power and Energy Engineering, 2, 718-728. http://dx.doi.org/10.4236/jpee.2014.24096

[4] Bletskan, D.I. (2004) Crystalline and Glassy Chalcogenides of Si, Ge, Sn and Alloys on Their Base. Vol. 1, Zakarpattia Uzhhorod.

[5] Bletskan, D.I. and Kabatsii, V.M. (2014) Fiber-Optical Sensor of Physical Values. Ukraine Patent No. 104813.

[6] Bletskan, D.I. and Kabatsii, V.M. (2014) Multi-Channel Optoelectronic Displacement Sensor. Ukraine Patent No. 105708.

[7] Bletskan, D.I. and Kabatsii, V.M. (2014) Optoelectronic Sensor. Ukraine Patent No. 105856.

[8] Bletskan, D.I., Kabatsii, V.M. and Bletskan, M.M. (2014) Photoelectric Tracking Sensor for a Rotary Module. Ukraine Patent No. 106142.

[9] Bletskan, D.I. and Kabatsii, V.M. (2014) Photoelectric Sensor for a Solar Power Module. Ukraine Patent No. 106143.

[10] Bletskan, D.I., Kabatsii, V.M. and Bletskan, M.M. (2015) Photovoltaic Sensor for the Guidance of Solar Batteries on the Sun. Proceedings of the 16th International Scientific-Practical Conference "Modern Information and Electronic Technologies”, Odessa, 25-29 May 2015, 228-229. 